\title{
How Anxiety Can Affect and Change Perceptual Process?
}

\author{
Ahmad Daghigh*, Gayathri Varma Nanagiri**
}

\section{ABSTRACT:}

What we have tried to express in this paper includes the nature of anxiety and perception separately and through that we have explained that anxiety can be considered an important component in perceptual process, though it has always been believed as a psychological and neurological problem.

Keywords: Anxiety, Perception, Stress, Stimuli

\section{INTRODUCTION}

Perception by definition is the process of organizing and interpreting our surrounding environment. This involves our five senses of sight, sound, smell, taste, and touch. We receive information of our surroundings called stimulus through these five senses, which is then processed through to our brain.

Anxiety disorders can undertake many forms, having multiple causes, acting together to create the neurological disorders. Psychological characteristics, Life experiences and genetic factors all have an important role to develop anxiety. What is common to all forms of anxiety disorders is an increased state of arousal or fear. As a whole, arousal or fear is a response of the nervous system to an external stimulus, so called as the stressor in this mentioned case. So in case of anxious individuals, there is no external stressor. Some signals are activated internally in the brains which trigger the stress response. To realize how these spontaneous signals are generated, it is important to know the correspondent structures in the brain. Researches involving brain imaging technology and petrochemical techniques have indicated that the amygdale and the hippocampus have important roles in generating stress responses. In this article, we will go to present a brief description about how perceptional system works, what is nature of anxiety and how it changes our perceptional aspects.

* Department of Psychology \& Parapsychology, Andhra University, Visakhapatnam, India

** Department of English language, IGNOU_Indira Gandhi National Open University, Visakhapatnam, India

(C) 2014, A Daghigh, G Nanagiri; licensee IJIP. This is an Open Access Research distributed under the terms of the Creative Commons Attribution License (http://creativecommons.org/licenses/by/2.0), which permits unrestricted use, distribution, and reproduction in any Medium, provided the original work is properly cited. 


\section{SENSUAL STIMULI:}

Each of the five perceptual senses functions at an individual level in processing the stimulus. Our eyes capture what we see and the visual is then processed through the retina using cones and rods. The light entering our eye triggers photochemical reaction in rods and cones in back of retina. The chemical reaction in turn activates bipolar cells. Bipolar cells then activate the ganglion cells, the axons of which converge to form the optic nerve. The optic nerve transmits information to the brain's visual cortex via the thalamus.

Similarly, our ears transform sound waves into nerve impulses that our brain interprets. This feat is achieved by the equally complex system where the outer ear funnels sound waves to the eardrum. The bones of the middle ear amplify and relay the eardrum's vibrations through the oval window into the fluid filled cochlea. The resulting pressure changes in the cochlear fluid cause the basilar membrane to ripple, bending the air cells on the surface. Hair cell movements trigger impulses at the base of the nerve cells, whose fibers converge to form auditory nerve, which sends neural messages to the thalamus and onto the auditory cortex.

Well, if we were to detail how our senses of smell, taste and touch perform their tasks, it would be equally appalling and miraculous. This bottom - up process from the basic impact of the stimulus onto our sensory organs to the interpretation of the stimuli by our brain is just one side of the entire perceptual process.

If we were to function this way, we would be introducing ourselves every time we saw a person, or creating food every time we felt hungry. I wonder if we would have ever evolved. Yet, we did. The reason being, in our perception of the world around us, our brain also works top - down (beginning in the brain) to process the stimuli received by our senses. This involves a cognitive process using our experiences and expectations already stored in our brain.

This two - way process of perceiving our surroundings helps us recognize familiar faces, objects, savor the smell of a home - cooked meal, enjoy the sound of music playing in our neighbour's house. Although we view sensation and perception separately to analyze and discuss them, they are actual parts of one continuous process. Sensory analysis begins at the entry level, with information flowing from the sensory receptors to the brain (bottom - up processing). And, analysis also begins with the brain and flows down, filtering information through our experiences and expectations to produce perceptions (top - down processing).

\section{Main factors in perception process:}

There are various factors affecting the top - down processing of our perception. We generally have a tendency to organize stimuli into coherent groups. To bring order and form to the basic sensations, our minds follow certain rules such as proximity, similarity, continuity, connectedness and closure for grouping. Gestalt psychologists (twentieth century German psychologists) proposed that in perception the whole may exceed the sum of its parts. For 


\section{How Anxiety Can Affect and Change Perceptual Process?}

example, combine sodium, a corrosive metal, with chlorine, a poisonous gas, and something very different emerges - table salt. Like - wise, a unique perceived form emerges from a stimulus components. They emphasized our tendency to integrate pieces of information into meaningful wholes.

Another very important factor to influence our perception is our own experience we build over our lifetime. Experience guides, sustains, and maintains the brain's neural organization. We retain the imprint of early sensory experiences far into the future. We form mental predispositions called perceptual sets that function as lens through which we perceive the world. Through experience we form concepts or "schemas" that organize and interpret unfamiliar information. Also, our senses interact with one another. To say, that they influence one another, as when the smell of food influences its taste. Being told that a wine costs $\$ 90$ rather than its real $\$ 10$ pricemakes an inexpensive wine taste better and triggers more activity in a brain area thatresponds to pleasant experiences. As happens with the painplacebo effect, the brain's thinking frontal lobes offer information that other brainregions act upon.

Our pre - existing schemas influence how we interpret ambiguous sensations with top - down processing. The surrounding context helps create expectations that guide our perceptions. Emotional context can colour our interpretation of other people's behaviors, as well as our own. Our experiences, assumptions and expectations may give us a perceptual set that greatly influences what we perceive.

\section{NATURE OF ANXIETY:}

Anxiety is both an emotion and a cognition. Freud's psychoanalytic theory proposed that, beginning in childhood, people repress intolerable impulses, ideas, and feelings and this submerged mental energy sometimes produces mystifying symptoms, such as anxiety. Anxiety is a part of everybody's life. We may feel anxious, when we talk to strangers or in front of a group, look down from a tall building, face a board of interviewers, or on the first date.

Under the circumstances our mental predisposition is filled with emotions such as fear, negative feelings of low self - esteem or lack of self - confidence. What we try to perceive thereafter, under the effect of such negative perceptual set, is bound to be skeptical. Fortunately for most of us we overcome anxiety soon after we start experiencing the situation. It might just turn out to be another normal experience stored in our memory for future reference. But if this anxiety becomes intense and persistent like having sweaty palms, breathlessness, a person is said to have a psychological disorder. Anxiety disorders are psychological disorders characterized by distressing persistent anxiety or maladaptive behaviours that reduce anxiety.

Anxiety disorders are distinguished by the intensity of the emotion itself and the intensity of the impact of such anxieties on a person's perception of his own self and his surroundings. People 


\section{How Anxiety Can Affect and Change Perceptual Process?}

with generalized anxiety disorder (GAD) feel persistently and uncontrollably tense and apprehensive, for no apparent reason. In the more extreme panic disorder, anxiety escalates into periodic episodes of intense dread. Those with a phobia may be irrationally afraid of a specific object or situation. Persistent and repetitive thoughts (obsessions) and actions (compulsions) characterize obsessive -compulsive disorder (OCD). Symptoms of post-traumatic stress disorder(PTSD) include four or more weeks of haunting memories, nightmares, social withdrawal, jumpy anxiety, and sleep problems following some traumatic and uncontrollable event.

In its own way, each of the above mentioned anxiety disorders, our perception and thereby the quality of our life. Perception is not merely a projection of the world onto our brain. Rather sensations are disassembled into information bits that the brain then reassembles into its own functional model of the external world. Our brain constructs our perception. When we know a thing, it's hard to mentally simulate what it's like not to know. For example, when a person is attacked by a fierce dog, he could later develop a fear for all dogs. When bad events happen unpredictably and uncontrollably anxiety often develops. In one survey, 58 percent of those with social phobia experienced their disorder after a traumatic event. Once we have formed a wrong idea about reality, we have more difficulty seeing the truth.

Research shows that anxiety could be in our biology. We humans seem biologically prepared to fear threats faced by our ancestors. Our phobias focus on such specific fears: spiders, snakes, and other animals; close spaces and heights; storms and darkness. Our modern fears can also have an evolutionary explanation. For example, a fear of flying may come from our biological predisposition (perceptual set) to fear confinement and heights. Just as our phobias focus on dangers faced by our ancestors, our compulsive acts typically exaggerate behaviours that contributed to our species' survival. Grooming gone wild becomes hair pulling, washing up becomes ritual hand washing, checking territorial boundaries becomes rechecking an already locked door.

Some people more than others seem predisposed to anxiety. Genes matter. Pair a traumatic event with a sensitive, high - strung temperament and the result may be a new phobia. Genes influence disorders by regulating neurotransmitters. Some studies point to an anxiety gene that affects brain levels of serotonin, a neurotransmitter that influences sleep and mood. Other studies implicate genes that regulate the neurotransmitter glutamate. With too much glutamate, the brain's alarm centers become overactive.

Generalized anxiety, panic attack, PTSD, and even obsessions and compulsions are also manifested biologically as an arousal of brain areas involved in impulse control and habitual behaviours. Brain scans of people with OCD reveal elevated activity in specific brain areas duringbehaviours such as compulsive hand washing, checking, ordering, or hoarding. Though it is clear that biology underlies anxiety, the biological perspective cannot by itself explain all aspects of anxiety disorders. 


\section{How Anxiety Can Affect and Change Perceptual Process?}

Anxiety disorders may be caused by medical factors, genetics, brain chemistry, environmental factors, substance abuse or a combination of these. Anxiety can be learned from experiences too. Through conditioning, the short list of naturally painful and frightening events can multiply into a long list of human fears. One such learning process which can contribute to anxiety is stimulus generalization, as in the example of a dog attack. All dogs are perceived to be dangerous. An anxiety for any dog in general develops.

Another learning process, reinforcement, helps maintain our phobias and compulsions after they arise. Avoiding or escaping the feared situation reduces anxiety, thus reinforcing the phobic behaviours. Feeling anxious or fearing a panic attack, a person may go inside and be reinforced by feeling calmer. Compulsive behaviours operate similarly. If washing your hands relieves your feeling of anxiety, you may wash your hands again when those feelings return. We may also learn fear (anxiety) by observing others' fear. Human parents transmit fears to their children.

There are instances of strained or even broken relations. People with GAD worry continually, and they are often jittery, agitated, and sleep deprived. Concentration is difficult, as attention switches from worry to worry, and their tension and apprehension may leak out through furrowed brows, twitching eyelids, trembling, perspiration, or fidgeting. One of the worst characteristics of this disorder is that the person cannot identify, and therefore cannot deal with or avoid, its cause. This could lead to constant frustration. GAD is often accompanied with depressed mood. Much of what we perceive comes not just from the world "out there" but also from what's behind our eyes and between our ears. We often perceive what we expect to see.

Anxiety is most often triggered by the stress in our lives. Usually anxiety is a response to outside forces, but it is possible that we make ourselves anxious with negative self - talk : a habit of always telling ourselves the worst will happen. We may at times be obsessed with senseless or offensive thoughts that will not go away. Obsessive thoughts and compulsive behaviours may interfere with everyday living and cause the person distress. The obsessive thoughts become so haunting, the compulsive rituals so senselessly time - consuming that effective functioning becomes impossible.

Some people experience intense fear of being scrutinized by others called social phobia. Those with social phobia avoid potentially embarrassing social situations, such as speaking up, eating out, or going to parties - or will sweat, tremble, or have diarrhea when doing so. Social phobia is as simple as shyness taken to an extreme. Have we all not experienced shyness in our lives? People who have experienced several panic attacks may come to fear the fear itself and avoid situations where the panic has struck before. Given such fear, people may avoid being outside the home, in a crowd, on a bus, or on an elevator.

Anxiety whether normally inherent or a learned disorder has a deep impact on our everyday functioning. The complaints of recurring haunting memories and nightmares, a numbed social withdrawal, jumpy anxiety, and insomnia - are typical of a post traumatic stress disorder. Symptoms reported by battle - scarred veterans, survivors of accidents, disasters, and violent and 
sexual assaults are all alike. Research indicates the greater one's emotional distress during a trauma, the higher the risk for post - traumatic symptoms. And the more frequent an assault experience, the more adverse the long - term outcomes tend to be.

Anxiety intensified hurts, at times leading to physical problems, such as high blood pressure, or hyper - tension. Ironically, we need o be thankful for occasional pain. Pain is our body's way of telling us something has gone wrong. Drawing our attention to an injury (external or internal), a traumatic experience in the past, pain orders us to change our behavior.

Take the instance of those rare people born without the ability to feel pain (lack of sense of touch, similar to blindness or deafness) may experience severe injury or even die before adulthood. Without the discomfort that makes us occasionally shift position, their joints fail from excess strain, and without the warnings of pain, the effects of unchecked infections and injuries accumulate. Our memories in part exist to protect us in future. So there is biological wisdom in not being able to forget our most emotional or traumatic experiences, our greatest embarrassments, our worst accidents, and our most horrid experiences.

Our brain does more than register information about the world. Perception is not just opening a shutter and letting a picture print itself on the brain. We constantly filter sensory information and infer perceptions that make sense to us. Mind matters!

Given an object, our brain computes its colour, size, distance, movement and other aspects relative to its context (surroundings). Perception is also governed by comparison. Anxiety plays a role in performing this comparison between the present circumstance/ place and the similar past experience in our memory. In moderation it helps us improve our performance in life and realize our dreams.

\section{CONCLUSION:}

Anxiety disorders show the ability of the brain to create reality in the mind of human being. Perception as we mentioned above is a multiple process which can be affected by anxiety. This way we can assume factor of anxiety as a very important element which can not only play its role as a malfunction in process of perception but also, we may define it as a secondary functional thing in the whole perceptual process. Perception is mostly considered as the primary step of cognition and it can be affected by anxiety in the brain when anxiety causes a disruption in information interpreting system of the brain.

\section{REFERENCES:}

1."Anxiety Disorders." National Institute of Mental Health, 2006 http://www.nimh.nih.gov/publicat/anxiety.cfm.

2. Psychology, from inquiry to understanding, Scott Lilienfeld, 2012.

3. Psychology in Modules, David G. Myers, 2011. 